Proyecciones

Vol. 19, $\mathrm{N}^{\circ}$ 1, pp. 19-25, May 2000

Universidad Católica del Norte

Antofagasta - Chile

\title{
ON THE HYPERBOLIC DIRICHLET TO NEUMANN FUNCTIONAL IN ABELIAN LIE GROUPS *
}

\author{
CLAUDIO CUEVAS \\ Universidad de la Frontera, Temuco - Chile
}

\begin{abstract}
We prove the injectivity of the linearization of the hyperbolic Dirichlet to Neumann functional in a "small" compact neighborhood of the identity element e of an abelian Lie group $G$, under some suitable transversality condition.
\end{abstract}

AMS subject classification : 35L20, 58G20.

${ }^{*}$ This work was partially supported by DIUFRO, IN-16/98. 


\section{Introduction and Statement of the Result}

Let $\bar{\Omega}$ be a compact manifold of dimension $n$ with smooth boundary $\partial \Omega$ and let $\operatorname{Met}(\bar{\Omega})$ denote the set of all Riemannian metrics $g$ on $\bar{\Omega}$.

We consider the ansitropic wave equation

$$
\begin{aligned}
\square_{g} u & =\frac{\partial^{2} u}{\partial t^{2}}-\Delta_{g} u=0 \text { in } \Omega \times(0, T), \\
u & =f \text { on } \Gamma=\partial \Omega \times(0, T), f \in C_{0}^{\infty}(\Gamma), \\
u & =\frac{\partial u}{\partial t}=0 \text { in } \Omega \times\{0\} .
\end{aligned}
$$

There is a unique solution to (1.1); hence we may define the hyperbolic Dirichlet to Neumann map as the linear operator $\Lambda_{g}: C_{0}^{\infty}(\Gamma) \longrightarrow$ $C^{\infty}(\Gamma)$, given by

$$
\Lambda_{g} f=\left.d u \cdot \nu_{g}\right|_{\Gamma}=\left.\frac{\partial u}{\partial \nu_{g}}\right|_{\Gamma}
$$

where $u$ is the unique solution to (1.1) and $v_{g}$ is the $g$-interior unit normal to $\partial \Omega$. The hyperbolic Dirichlet to Neumann Functional:

$$
\begin{aligned}
\Lambda: \operatorname{Met}(\bar{\Omega}) & \longrightarrow \mathrm{O}_{p}(\Gamma) \\
g & \mapsto \Lambda_{g},
\end{aligned}
$$

where $\mathrm{O}_{p}(\Gamma)$ denotes the space of all linear operators from $C_{0}^{\infty}(\Gamma)$ into $C^{\infty}(\Gamma)$, is known to be invariantly defined on the orbit obtained by the action over $\operatorname{Met}(\bar{\Omega})$ of the group $\mathcal{D}$ of all diffeomorphism $\psi$ of $\bar{\Omega}$, each of which restricts to the identity on $\partial \Omega$. A natural conjecture is that this is the only obstruction to the uniqueness of $\Lambda$. From the point of view of applications, an even more important problem is to give a method to reconstruct $g$ from $\Lambda_{g}$.

The elliptic Dirichlet to Neumann map was treated by several authors and is closely related to the physical problem referred as Electrical Impedance Tomography, of determining the conductivity of a body from measurement of voltage potential and corresponding current fluxes at the boundary (see [4]).

For fixed $g$, we consider the following map:

$$
\psi \in \mathcal{D} \stackrel{A_{g}}{\longrightarrow} \psi_{g}^{*} \in \operatorname{Met}(\bar{\Omega})
$$


It is easy to see that the tangent space $T_{I} \mathcal{D}$ of $\mathcal{D}$ at the identity mapping $I$ is the vector space $\Gamma_{0}(T \bar{\Omega})$ of all smooth vector fields on $\bar{\Omega}$ which vanish on $\partial \Omega$. On the other hand, the tangent space $T_{g}(\operatorname{Met}(\bar{\Omega}))$ of $\operatorname{Met}(\bar{\Omega})$ at $g$ is the vector space $\Gamma\left(S^{2} \bar{\Omega}\right)$ of all smooth sections of symmetric 2 -tensors on $\bar{\Omega}$. We introduce on the spaces $\Gamma_{0}(T \bar{\Omega})$ and $\Gamma\left(S^{2} \bar{\Omega}\right)$ the inner products

$$
\begin{aligned}
& \langle X, Y\rangle=\int_{\bar{\Omega}} g(X, Y) v_{g}, \quad X, Y \in \Gamma_{0}(T \bar{\Omega}), \\
& \langle\langle m, l\rangle\rangle=\frac{1}{n} \int_{\bar{\Omega}} \operatorname{tr}(\hat{m} \circ \hat{l}) v_{g}, \quad m, l \in \Gamma\left(S^{2} \bar{\Omega}\right),
\end{aligned}
$$

where $v_{g}$ (resp., $t r$ ), denote the volume element (resp., the trace) associated to $g$ and $\hat{m}$ is the unique linear map defined by

$$
g(\hat{m} u, v)=m(u, v) \text { for all } u, v \in \Gamma(T \bar{\Omega}) .
$$

Considerer as in [3], the formal linearizations of $A_{g}$ at $I$ and of $\Lambda$ at $g$, respectively :

$$
A_{g}^{\prime}[I]:=A_{g}^{\prime}: \Gamma_{0}(T \bar{\Omega}) \longrightarrow \Gamma\left(S^{2} \bar{\Omega}\right)
$$

and

$$
\Lambda_{g}^{\prime}: \Gamma\left(S^{2} \bar{\Omega}\right) \longrightarrow \mathrm{O}_{p}(\Gamma) .
$$

Let $\left(A_{g}^{\prime}\right)^{*}$ denote the formal adjoint of $A_{g}^{\prime}$ with respect to the inner products (1.5) and (1.6) and $\operatorname{diam}_{g}(\Omega)$ the diameter of $\Omega$ in the metric $g$. In [3], the authors stated the following :

Conjecture 1.1 Let $\Omega_{0} \subset \Omega$ be a submanifold, $m \in \Gamma\left(S^{2} \bar{\Omega}\right)$ have support in $\bar{\Omega}_{0}$ i.e., $m \in \Gamma_{0}\left(S^{2} \bar{\Omega}\right)$ and assume that a) $\Lambda_{q}^{\prime}(m)=0$, b) $\left(A_{g}^{\prime}\right)^{*}(m)=0$ and $\left.c\right) \operatorname{diam}_{g}\left(\Omega_{0}\right)<T$ is sufficiently small that the exponential map for $g$ is a global diffeomorphism. Then $m$ is identically zero.

As in [3] we refer to condition b) as the Transversality Condition. We remind that condition c) is necessary to avoid the appearance of caustics. The Tranversality Condition replace the harmonic hypothese used in [4]. 
The main results of this paper is:

Theorem 1.1 Conjecture 1.1 holds if $G$ be an abelian Lie group and $\bar{\Omega}$ is a compact neighborhood of $G$ at the identity element $e$, and $g$ is an invariant metric on $G$ and $m \in \Gamma_{0}\left(S^{2} \bar{\Omega}\right)$.

We are able to obtain an generalization Cardozo-Mendoza Theorem 1.1 [3] as corollary of Theorem 1.1.

Corollary 1.1 Conjecture 1.1 holds if $\bar{\Omega}$ is a bounded domain of $\mathbb{R}^{\mathrm{n}}$, and $g$ is a metric on $\mathbb{R}^{\mathrm{n}}$ and $m \in \Gamma_{0}\left(S^{2} \bar{\Omega}\right)$.

Corollary 1.2 Conjecture 1.1 holds if $\bar{\Omega}$ is a bounded domain of the Torus $T^{n}$, and $g$ is an invariant metric on $T^{n}$ and $m \in \Gamma_{0}\left(S^{2} \bar{\Omega}\right)$.

Corollary 1.3 Conjecture 1.1 holds if $\bar{\Omega}$ is a bounded domain of the product $\mathbb{R}^{\mathrm{n}} \times \mathrm{T}^{\mathrm{m}}$ and $g$ is an invariant metric on $\mathbb{R}^{\mathrm{n}} \times \mathrm{T}^{\mathrm{m}}$ and $m \in$ $\Gamma_{0}\left(S^{2} \bar{\Omega}\right)$.

Remark 1.1 In [3], the authors proved that Conjecture 1.1 holds if $\bar{\Omega}$ is a bounded domain of $\mathbb{R}^{\mathrm{n}}, \mathrm{n} \geq 2$ and $g$ is the Euclidean metric. They also proved the conjecture when $n=2$ and $g$ is near the Euclidean metric in the $C^{3}$-topology. In [1] they prove that Conjecture 1.1 holds if $\bar{\Omega}$ is a bounded domain of the hyperbolic space (resp.,n-sphere) and $g$ is the canonical metric in this spaces. In [2], the authors proved the uniqueness conjecture for the case when the manifold is a sufficiently small bounded domain of $\mathbb{R}^{3}$, under suitable geometric conditions and the metric $g$ is $C^{3}$-close to Euclidean metric. We shall make use of the invariant formulas for $A_{g}^{\prime},\left(A_{g}^{\prime}\right)^{*}$ and $\Lambda_{g}^{\prime}$ proved in Section 2 [3].

\section{Proof of Theorem 1.1}

Let $G$ be an abelian Lie group, and $\operatorname{Lie}(G)$ the algebra formed by the set of all left invariant vector fields on $G$, and $\bar{\Omega}$ is a compact neighborhood of $G$ at the identity element $e$, and $\Gamma_{0}\left(S^{2} \bar{\Omega}\right)$ denote the vector space of all smooth sections of symmetric 2 -tensors on $G$ which are supported on $\bar{\Omega}$. 
Proof of Theorem 1.1 Let $x \in \Omega, v \in \operatorname{Lie}(G)$. The geodesic with initial tangent vector $(x, v) \in T G \cong G \times \operatorname{Lie}(G)$ is given by $\gamma(t)=x \exp (t v)$. Since $x \in \Omega$ there exists an element $A \in \operatorname{Lie}(G)$ such that $x=\exp (A)$.

Let $\left\{E_{\mu}\right\}_{\mu=1, \ldots, n}$ a orthonormal frame field in $\Gamma(T G)$ then we can define a orthonormal system $\left\{\tilde{E}_{\mu}, V_{\mu}\right\}_{\mu=1, \ldots, n}$ in $\Gamma(T T G)$ as follows. We consider the diagram

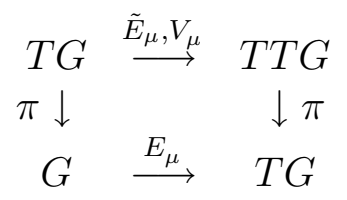

where $\tilde{E}_{\mu}(x, v)=\left(x, v, E_{\mu}, 0\right)$ and $V_{\mu}(x, v)=\left(x, v, 0, E_{\mu}.\right)$

Let $x=\left(x_{1}, \ldots, x_{n}\right)$ the canonical coordinates system (of the first kind) of $G$ around the identity $e$ associated to the orthonormal frame field $\left\{E_{\mu}\right\}_{\mu=1, \ldots, n}$ and $v=\left(v_{1}, \ldots, v_{n}\right)$ the canonical coordinates of $\operatorname{Lie}(G)$.

Now we consider the function $F_{t}: T G \longrightarrow \mathbb{R}$ defined by

$$
F_{t}(x, v)=m(x \exp (t v))(v, v)
$$

and the operator

$$
\mathcal{L}=\sum_{\mu=1}^{n} \tilde{E}_{\mu} V_{\mu} \in \operatorname{End}\left(C^{\infty}(T G)\right)
$$

We obtain

$$
\mathcal{L} F_{t}=(-t) \Delta_{g} F_{t}+2 \sum_{\mu, \beta=1}^{n} v_{\mu} E_{\beta} m_{\mu \beta}(\gamma),
$$

where $\Delta_{g}$ denotes the Laplace-Beltrami operator associated to the metric $g$ and $m_{\mu \beta}=m\left(E_{\mu}, E_{\beta}\right)$.

On the other hand, the Transversality Condition satisfied by $m$ means that

$$
E_{1}\left(m_{\mu 1}\right)+\ldots+E_{n}\left(m_{\mu n}\right)=0, \quad \mu=1, \ldots, n
$$

Using (2.1) and (2.2) we conclude that 


$$
\mathcal{L} F_{t}=(-t) \Delta_{g} F_{t}
$$

We define

$$
H^{k}(x, v)=\int_{-\infty}^{\infty}(-t)^{k} F_{t}(x, v) d t
$$

for all $k=0,1, \ldots$

Using (2.3), it is easy to show that

$$
\mathcal{L} H^{k}=\Delta_{g} H^{k+1}, \quad k=0,1,2, \ldots
$$

If we fix $v$, since $m$ is supported in $\bar{\Omega}$, it follows that $H^{k}(\cdot, v)$ for all $k$ $=1,2, \ldots$ vanish on a non-empty open subset of $G$. From Proposition 3.1 in [3], $H^{0}=0$ and hence $H^{1}$, and in fact all $H^{k}$ are harmonic functions. Therefore $H^{k}$ is identically zero for all $k=0,1,2, \ldots$.

Observe that $t \rightarrow F_{t}$ is supported in some closed interval $[a, b]$ containing zero. Since the subalgebra generated by the family of functions $\left\{(-t)^{k}\right\}_{k=0.1 \ldots}$ is dense in $C([a, b], \mathbb{R})$, we obtain that $t \rightarrow F_{t}$ is identically zero. If we take $t=0$ and recall that $m$ is symmetric, we conclude that $m$ is identically zero.

\section{References}

1. Cardoso, F. and Cuevas, C., "On the Hyperbolic Dirichlet to Neumann functional in $H^{n}$ and $S^{n}$ ", Proyecciones Vol. 17, $\mathrm{N}^{o}$ 1, pp. $63-70,(1998)$.

2. Cardoso, F. and Cuevas, C., "On the Hyperbolic Dirichlet to Neumann functional', to appear Portugaliae Mathematica. Vol. $56, \mathrm{~N}^{o} 4,(1999)$

3. Cardoso, F. and Mendoza, R., "On the Hyperbolic Dirichlet to Neumann functional', Comm. in Partial Diff. Equations, 21, pp. $1235-1252,(1996)$.

4. Uhlmann, G., "Inverse boundary value problems and applications". Astérisque, 207, pp. 153 - 211, (1992). 
Received : April 10, 1999.

\section{Claudio Cuevas}

Departamento de Matemáticas

Facultad de Ingeniería

Universidad de la Frontera

Casilla 54-D

Temuco

Chile 